\title{
Nonlinear Estimation of Quadcopter States Using Unscented Kalman Filter
}

\author{
Ahmed Abdulmahdi Abdulkareem Alawsi ${ }^{1}$, Basil H Jasim ${ }^{2}$, Safanah Mudheher Raafat ${ }^{3}$ \\ ${ }^{1}$ College of Science, Physics Dept., University of Wasit, Wasit Iraq \\ ${ }^{2}$ Electrical Engineering Dept., University of Basra, Basra Iraq \\ ${ }^{3}$ Control and System Engineering Dept., University of Technology, Baghdad Iraq;
}

\begin{abstract}
Article Info
Received

\section{Keyword:}

Quadcopter

PID controller

Autonomous flight

Delivery by quadcopter

Sensor noise of quadcopter,

UKF

EKF

ABSTRACT

In recent years, using Unmanned Arial Vehicles (UAV) like quadcopter in civilian and military fields are increased dramatically. Performance and robustness are the most important specifications required for most applications. Different sensors are usually used for a quadcopter to provide the necessary measured states (attitude and position) for control. The white noise generated by physical sensors is one of the important issues that affect the quality of states measurements. The available solutions are still have limited performance for a wide range of nonlinearity. In this paper, Unscented Kalman Filter (UKF) is proposed as a robust estimator that has the ability to work efficiently with high nonlinear systems. Modified PID (PI-D) controller which has better properties than traditional PID controller is used with proposed filter in order to get better performance of quadcopter. The obtained results are compared with that of Extended Kalman Filter (EKF) and proved to be more reliable. Moreover, the results show that the proposed filter largely decreases the error generated by noise and improves the performance of quadcopter better than the EKF.
\end{abstract}

\section{Corresponding Author:}

Second Author,

Departement of Electrical Engineering,

Basrah University,

Basrah, Iraq

Email: hanbas632@gmail.com

\section{Introduction}

The use of the unmanned aerial vehicles (UAV), such as the quadcopter, has recently increased in various civilian areas because of its advantages, particularly vertical takeoff, landing in a small area and hovering. Therefore, the quadcopters have become the focus of great attention by researchers to find scientific and practical solutions to the problems that arise when using it in various applications such as agriculture, monitoring, rescue missions, delivery applications, etc. [1]. Quadcopter is dynamically unstable and require on board a control algorithm to control its attitude and position through flight. A widespread controller is Modified PID (PI-D) controller used to control the quadcopter because its simplicity calculations and easy of implement in real time. Also, sensors such as gyroscope, accelerometer, GPS, compass, barometer are required to fed control algorithm with measured states to keep quadcopter under control [2]. Most of the sensors used in quadcopters are cheap (low quality) because the high quality devices are very expensive and the price exceeds the price of the quadcopter itself [3]. Therefore, the information signals measured by these sensors have much noise caused by sensors itself and uncertainties in measurements of control algorithms [4]. 
Noise has a negative effect on the control algorithm and this leads to decreasing the performance and robustness of the quadcopter [5]. To improve these two factors, a reliable states estimation algorithm should be added to the controller to estimate accurate states. Several methods have been proposed to improve the performance of quadcopter. The main progress in previous attempts can be summarized as follows: In [3], this approach uses Kalman Filter (KF) to estimate actual states of quadcopter from noisy measured states. The KF is designed especially for linear systems while the quadcopter is nonlinear system. Therefore, this filter does not work properly in the nonlinear behavior of quadcopter and this lead to push the quadcopter out of control, while in the approach of [5], the authors uses Extended Kalman Filter (EKF) to estimate actual states of quadcopter from noisy measured states. EKF is a modified version of KF designed to operate properly with nonlinear systems. The EKF uses linearized model of nonlinear system to estimate its states. Therefore, its stability is highly affected by nonlinearities of the system. Moreover, the EKF has a relatively slower performance since it takes more iterations to estimate actual states [6].

Consequently, the quadcopter performance and robustness directly affected by estimated states. Therefore, improving the quality of estimation leads to increasing the stability of quadcopter against external influences like wind or other disturbances. In this paper, Unscented Kalman Filter (UKF) is proposed as states estimator to estimate actual states of quadcopter from measured noisy states. UKF is designed to operate properly with nonlinear system because it uses multiple points called Sigma Points to describe the behavior of nonlinear system through unscented transform algorithm. Therefore, the states being estimated by UKF are highly accurate. Also, UKF is faster than EKF because it less mathematical operations complexity to estimate actual states $[6,7]$.

Matlab simulink ver. R2018a is used to test the effect of proposed filter on the quadcopter control algorithm. After several iterations by matlab simulink platform, the results show that UKF improves the performance of quadcopter better than EKF. Also, movements of quadcopter become smoother and closer to the desired path. Therefore, the proposed filter is also improving the resistance of quadcopter to the external disturbances like wind.

The rest of paper is organized as follows: Section 2 describes the dynamic model of the quadcopter, Section 3 describes altitude and attitude controls by modified PID controller, Section 4 gives the basics of UKF, Section 5 gives a detailed description of block diagram of proposed control method, Section 6 gives the results and discussion, and the conclusion is given in section 7 .

\section{Dynamic Model of Quadcopter}

Mathematical dynamical model of quadcopter is needed to study its stability, design suitable controller and implement future modifications and developments. Generally, the Quadcopter use two pairs of identical fixed pitched propellers as shown in figure 1; two clockwise (CW) and two counterclockwise (CCW). Figure 1 shows most of the forces that affect the quadcopter. Some of these forces are external forces such as gravity and the other are generated by the quadcopter through its motors in order to enable it to control, fly and perform the missions $[8,9]$.

There are several papers, articles, and books showed how to drive the mathematical model of quadcopter. In this section, the mathematical dynamics equations of the quadcopter are mentioned and briefly explained so for more details, please see the relevant sources. The quadcopter has six degree of freedom in terms of linear position $\xi=(\mathrm{x}, \mathrm{y}, \mathrm{z})$ and the attitude (angular position) which is defined by Euler angles $\mathrm{H}=$ (roll $(\phi)$, pitch $(\Theta)$, yaw $(\psi)$ ) with respect to the inertial frame. The Euler angles determine the rotation of the quadcopter around each axis. Roll angle $\phi$ determines the rotation around $x$-axis while Pitch angle $\Theta$ determines the rotation around y-axis and yaw angle $\psi$ determines the rotation around z-axis [10-13]. 


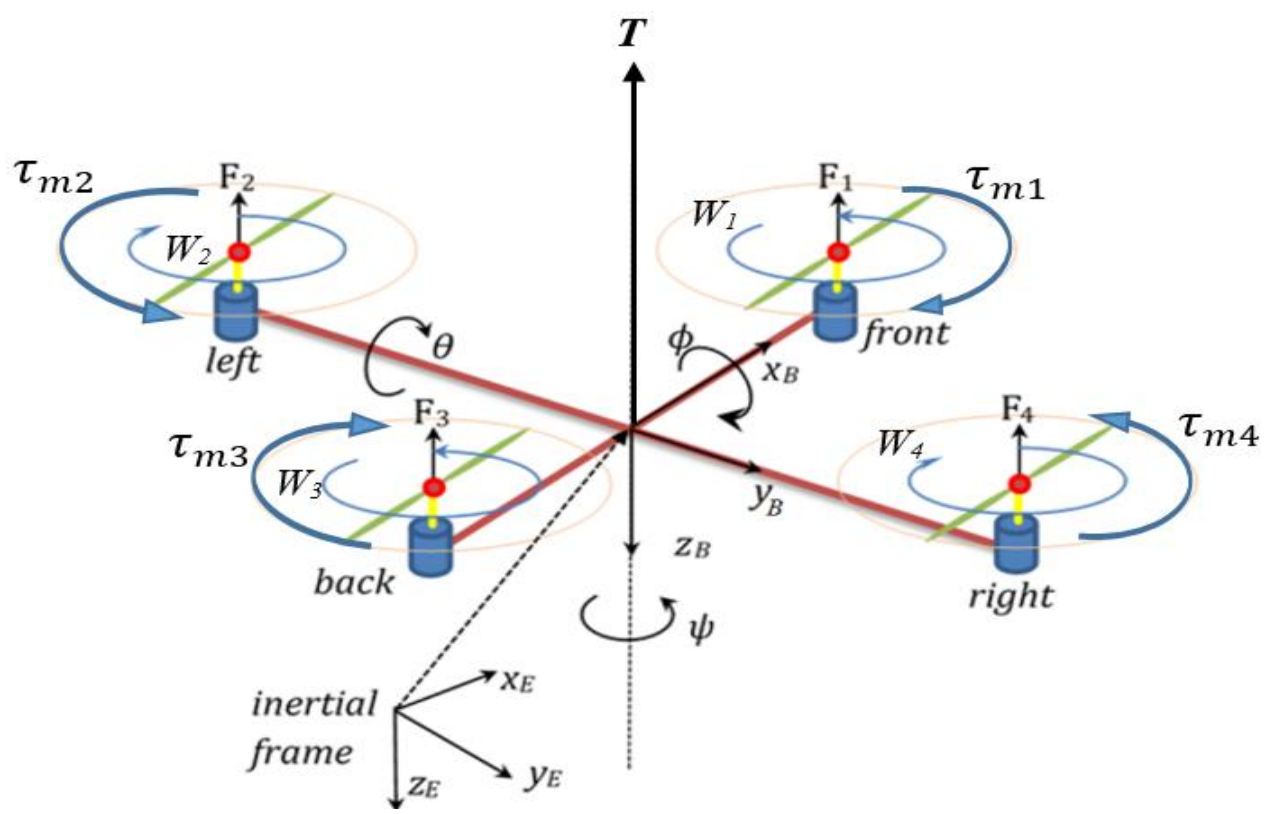

Figure 1. The Inertial and Body Frames of the quadcopter [14]

The movement of the quadcopter is always represented by linear and angular acceleration equations. Equations 1 represents the angular mathematical dynamic equations which are used to measure the angular states (angles) of quadcopter around $\mathrm{x}$-axis, $\mathrm{y}$-axis, and z-axis [10-13].

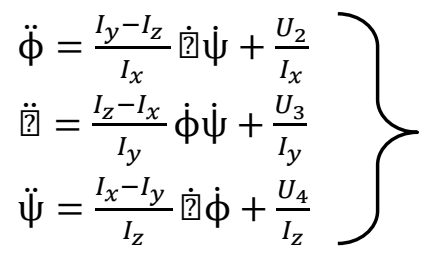

where, $U_{2}, U_{3}$ and $U_{4}$ are control inputs generated by controller which are used to generate the required torques $\tau_{\phi}, \tau_{\square}$ and $\tau_{\psi}$ by quadcopter motors to move it around linear axis (x, y, and z). Ix, Iy, and Iz are the moments of inertia about the $\mathrm{x}, \mathrm{y}$, and $\mathrm{z}$-axes, respectively [10-13].

Equation 2 represents the linear motion in the direction of $\mathrm{x}, \mathrm{y}$, and z-axis and it is used to measure the linear states of quadcopter.

$$
\left.\begin{array}{l}
\ddot{x}=\frac{U 1}{m}\left(c_{\Psi} s_{\square} c_{\phi}+s_{\Psi} s_{\phi}\right) \\
\ddot{y}=\frac{U 1}{m}\left(s_{\Psi} s_{\square} c_{\phi}-c_{\Psi} s_{\phi}\right) \\
\ddot{z}=\frac{U 1}{m} c_{\square} c_{\phi}-g
\end{array}\right\}
$$

where, $U 1$ is the control input generated by flight controller to introduce the desired thrust force for quadcopter. $m$ represents mass of the quadcopter. $c_{\phi}=\cos (\phi), s_{\phi}=\sin (\phi), c_{\square}=\cos ($ 回 $), s_{\square}=\sin ($ 回 $) . \mathrm{g}$ is the earth's gravity [10-13].

Equations 1 and 2 can be used with special platform like Matlab simulink to test the proposed method. The measured states $(x, \dot{x}, y, \dot{y}, z, \dot{z}, \phi, \dot{\phi}, \theta, \dot{\theta}, \Psi, \dot{\psi})$ of quadcopter are obtained by performing first and second integrations to the equations 1 and 2 .

The state space of quadcopter mathematical model is representing the state transition function as in the following equations [15] 


$$
\left[\begin{array}{c}
\dot{x}_{1} \\
\dot{x}_{2} \\
\dot{x}_{3} \\
\dot{x}_{4} \\
\dot{x}_{5} \\
\dot{x}_{6} \\
\dot{x}_{7} \\
\dot{x}_{8} \\
\dot{x}_{9} \\
\dot{x}_{10} \\
\dot{x}_{11} \\
\dot{x}_{12}
\end{array}\right]=\left[\begin{array}{c}
x_{2} \\
(U 1 / m) *\left(\cos \left(x_{7}\right) \sin \left(x_{9}\right) \cos \left(x_{11}\right)+\sin \left(x_{7}\right) \sin \left(x_{11}\right)\right) \\
x_{4} \\
(U 1 / m) *\left(\cos \left(x_{7}\right) \sin \left(x_{9}\right) \sin \left(x_{11}\right)-\sin \left(x_{7}\right) \cos \left(x_{11}\right)\right) \\
x_{6} \\
\left((U 1 / m) *\left(\cos \left(x_{7}\right) \cos \left(x_{9}\right)\right)\right)-g \\
x_{8} \\
U 2 / I_{X X} \\
x_{10} \\
U 3 / I_{y y} \\
x_{12} \\
U 4 / I_{z z}
\end{array}\right]
$$

Also, the measurement functions are representing the measured states $\left(x_{2}, x_{4}, x_{6}, x_{8}, x_{10}\right.$, and $\left.x_{12}\right)$ in the equation 3 and can be expressed as in equation (4):

$$
\left[\begin{array}{c}
x=\dot{x}_{1}=x_{2} \\
y=\dot{x}_{3}=x_{4} \\
z=\dot{x}_{5}=x_{6} \\
\phi=\dot{x}_{7}=x_{8} \\
?=\dot{x}_{9}=x_{10} \\
\psi=\dot{x}_{11}=x_{12}
\end{array}\right]
$$

Equations (3) and (4) are used by UKF block to estimate accurate states of quadcopter.

\section{Modified PID controller}

The quadcopter is aerodynamically unstable and therefore a control system is needed to control its attitude and position to keep it stable during flight. The modified PID (PI-D) controller as shown in figure 2 is used in this paper because it is simple, efficient, and easy to implement in real and simulation platforms [16]. The main task of PI-D controller is to control the quadcopter through flight and hovering by decreasing the error between desired and measured states.

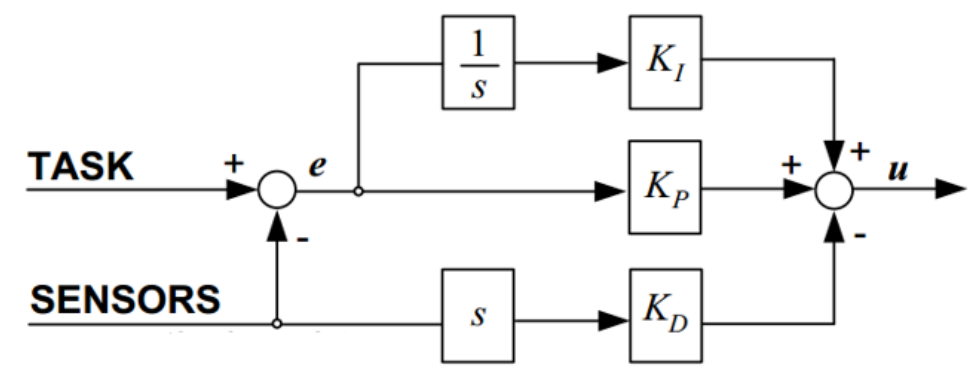

Figure 2. Modified PID (PI-D) controller structure

The mathematical representation of P-ID controller is [15-18]:

where,

$$
P I D(e)=k_{p} e(t)+k_{I} \int_{0}^{t} e(\tau) d \tau-k_{D} \frac{d}{d t} \text { state }
$$

$e(t)=$ desired state $(\mathrm{t})-$ measured state $(\mathrm{t})$

$k_{p}, k_{I}$ and $k_{D}$ are the proportional, integral and derivative gains respectively. The $\frac{d}{d t}$ state represents the derivative of state measured by sensor.

The desired control signals can be generated using equation 5 as follows:

$$
U_{j}=k_{p_{h}} \boldsymbol{e}_{\boldsymbol{h}}+k_{I_{h}} \int \boldsymbol{e}_{\boldsymbol{h}}-k_{D_{h}} \dot{h}_{\text {meas }}+m g
$$


where, $j=[1, \ldots, 4], h=[z, \phi$, 目, $\psi]$ and $e_{h}$ is the error signal, i.e. the difference between desired and measured states $\left(e_{h}=h_{\text {des }}-h_{\text {meas }}\right)$, where $h_{\text {des }}$ and $h_{\text {meas }}$ are desired and measured values respectively. The control signal $U 1$ is used to control the altitude of quadcopter while $U 2, U 3$, and $U 4$ are control signals used to control the three angles Roll, Pitch, and Yaw of quadcopter. The mass of quadcopter is represented by $\mathrm{m}$ where $g=9.81 \mathrm{~m} / \mathrm{s}^{2}$ is the earth gravity.

The quadcopter has 6 DOF and only 4 actuators (motors) so it is not possible to control all these DOF directly. The control equations (6) are used to directly control 4 DOF $(\mathrm{z}, \phi$, ?, $\psi 4)$. The roll and pitch angles make the quadcopter move towards the desired $\mathrm{x}$ and $\mathrm{y}$. So, the desired $\mathrm{x}$ and $\mathrm{y}$ will be used first to calculate the desired roll and pitch angles to be able to control $\mathrm{x}$ and $\mathrm{y}$ positions indirectly. The equations which are used to calculate desired roll and pitch angles are frequently written in terms of desired $\mathrm{x}$ and $\mathrm{y}$ accelerations. The following equations are used to calculate desired acceleration though desired $\mathrm{x}, \mathrm{y}$ and $\mathrm{z}$ [13]:

$$
\begin{aligned}
& \ddot{x}_{\text {des }}=\left(k_{p_{x}} e_{x}+k_{I_{x}} \int e_{x}-k_{D_{x}} \dot{x}_{\text {meas }}\right) / m \\
& \ddot{y}_{\text {des }}=\left(k_{p_{y}} e_{y}+k_{I_{y}} \int e_{y}-k_{D_{y}} \dot{y}_{\text {meas }}\right) / m \\
& \ddot{z}_{\text {des }}=\left(k_{p_{z}} e_{z}+k_{I_{z}} \int e_{z}-k_{D_{z}} \dot{z}_{\text {meas }}-m g\right) / m
\end{aligned}
$$

The equations below represent the relations between desired linear accelerations with respect to the inertial frame, and the desired related angles roll, pitch and yaw with respect to the body frame [13]:

$$
\begin{aligned}
& \phi_{d e s}=\sin ^{-1}\left(\frac{\ddot{x}_{\text {des }} s_{\Psi}-\ddot{y}_{\text {des }} c_{\psi}}{\sqrt{\ddot{x}_{d e s}^{2}+\ddot{y}_{d e s}^{2}+\left(\ddot{z}_{d e s}+g\right)^{2}}}\right) \\
& \text { ? }_{d e s}=\tan ^{-1}\left(\frac{\ddot{x}_{\text {des }} c_{\Psi}-\ddot{y}_{\text {des }} s_{\Psi}}{\ddot{z}_{\text {des }}+g}\right)
\end{aligned}
$$

The block diagram of the quadcopter with a PI-D controller is as shown in Figure 3 [13].

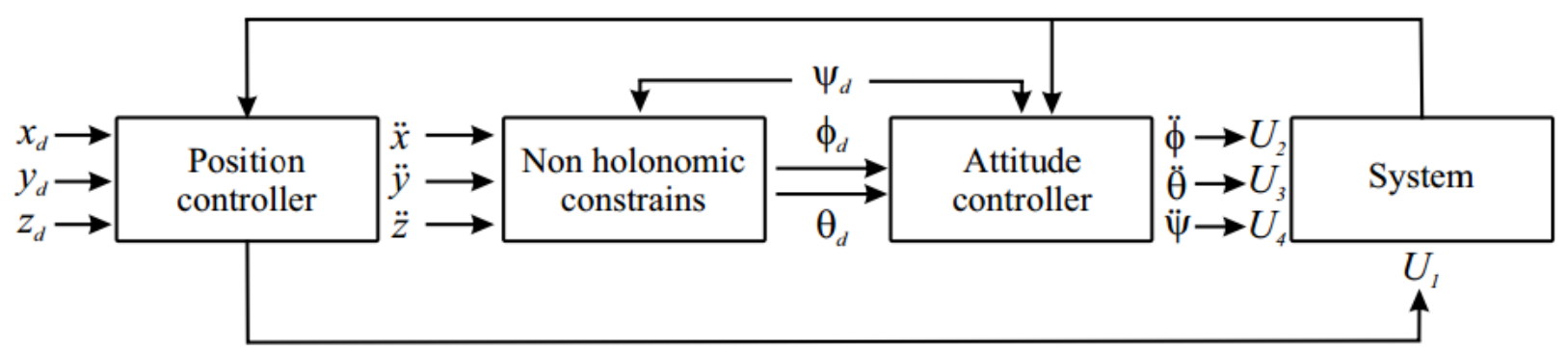

Figure 3. Block diagram of the control algorithm of quadcopter with PI-D controller

\section{Unscented Kalman Filter (UKF)}

The Unscented Kalman Filter (UKF) is a modern nonlinear filter used to estimate the accurate states of nonlinear systems corrupted by Gaussian noise. UKF is an efficient method to calculate the statistics of a random variable by a nonlinear transformation called Unscented transformation (UT). UKF is based on a simple assumption that it is easier to approximate a Gaussian distribution than to approximate an arbitrary nonlinear function or transformation. A finite number of points called sigma points are generated when applied the UKF to the measured data, to sample the prior distribution, and keeps the sample mean and sample covariance the same as the original distribution [19], [20].

The UKF has two phases: the time-update (prediction phase) and the measurement-update (correction phase). In the prediction phase, sigma points are calculated for the last available estimated states and each point is assigned a weight. Thereafter, the sigma points are put through the nonlinear model equations to obtain predicted states. In the measurements-update phase, the predicted states are corrected by adding the effect of the information obtained from the new state measurements to estimate the actual states [19], [21]. 
UKF predicts the states of the last estimated states by the following equation:

$$
\tilde{X}_{k+1 \mid k}=\phi_{k+1 \mid k}\left[X_{k \mid k}\right]+G_{k}
$$

The discrete predicted states $\tilde{X}_{k+1 \mid k}$ at $\mathrm{k}+1$ are updated with the measurement information by the following equation:

$$
\widehat{X}_{k+1 \mid k}=\mathrm{H}_{k+1 \mid k}\left[\tilde{X}_{k+1 \mid k}\right]+V_{k+1}
$$

where $\phi_{k+1 \mid k}$ is the discrete state transition matrix from $\mathrm{k}$ to $\mathrm{k}+1, X_{k \mid k}$ is estimated states at $\mathrm{k}, G_{k}$ is the process noise vector at $\mathrm{k}, \hat{X}_{k+1}$ is the estimated state vector at $\mathrm{k}+1, V_{k+1}$ is the measurement noise vector, $\mathrm{H}_{k+1 \mid k}$ is the observation matrix. The state transition matrix is linear and derived from dynamic model acceleration equations of the quadcopter. Only the observation matrix $\mathrm{H}_{k+1 \mid k}$ contains nonlinear equations and it is calculated by the following equation:

$$
\mathrm{H}_{k+1 \mid k}=\sum_{i=0}^{2 * n} W_{i} * H\left(X_{i, k+1 \mid k}^{\tilde{x}}\right)+X_{i, k+1}^{V}
$$

where, $W_{i}$ are the weights, $X_{i, k+1 \mid k}^{\tilde{x}}$ are the sigma points which describe the predicted states $\tilde{X}_{k+1 \mid k}, X_{i, k+1}^{V}$ are the sigma points describing the measurement noise [19-22].

The number of sigma points depends on the dimensionality of the system. The general formula to calculate the number of sigma points is $2 n+1$, where $n$ is the dimension of the system.

The following equations are used to calculate the sigma points for the predicted states $\tilde{X}_{k+1 \mid k}$ [19], [21]:

$$
\begin{array}{ll}
X_{0}=\overline{\tilde{X}} & \\
X_{i}=\overline{\tilde{X}}+\left(\sqrt{(n+\lambda) p_{\tilde{x} \tilde{x}}}\right)_{i} & \mathrm{i}=1, \ldots \ldots, \mathrm{n} \\
X_{i+n}=\overline{\tilde{X}}+\left(\sqrt{(n+\lambda) p_{\tilde{x} \tilde{x}}}\right)_{i} & \mathrm{i}=\mathrm{n}+1, \ldots \ldots, 2 \mathrm{n}
\end{array}
$$

where, $\overline{\tilde{X}}$ is the mean of the predicted states $\tilde{X}_{k+1 \mid k}, \lambda$ is the scaling factor which tells how far from the mean we should choose our sigma points, $p_{\tilde{x} \tilde{x}}$ is the covariance matrix of predicted states, $\left(\sqrt{(n+\lambda) p_{\tilde{x} \tilde{x}}}\right)_{i}$ is the ith column of the square root weighted covariance [19].

\section{Quadrotor States Estimation Using UKF}

The UKF is implemented in this paper to estimate accurate linear and angular states from noisy measured states of quadcopter. The proposed filter is one of the robust and reliable nonlinear estimator. The simulations are performed using Matlab Simulink to test the proposed method. Figure 4 shows the simulink block diagram of quadcopter with added white noise and UKF as estimation filter. In the position controller block, UI control signal is generated and the linear position $(\mathrm{x}, \mathrm{y}, \mathrm{z})$ is controlled in terms of desired linear acceleration using equations $(8,9$, and 10$)$.

In the non-holonomic constraints block, the desired Roll $\left(\phi_{d e s}\right)$ and Pitch ( $\left.{ }_{\text {des }}\right)$ angles are calculated with respect to the desired linear position $\left(X_{\text {des }}\right.$ and $\left.Y_{\text {des }}\right)$ using equations (11, and 12). In the angular position controller block, the equations $(5,6$, and 7$)$ are performed to generate the control signals $U 2, U 3$, and $U 4$ which are used to control the angular position of quadcopter. The control signals $(U 1, U 2, U 3$, and $U 4)$ are feeds to the plant block were dynamic equations 1 and 2 are included to measure linear and angular states of quadcopter. 
These measured states are equivalent to the measured states by sensors in real quadcopter and the noise is added to each measured states to make the simulation similar to reality. The UKF is used to decrease the effect of noise as much as possible and estimate optimal states to improve the performance of quadcopter. Now, the estimated states are feeds to the controller blocks as feedback signals to control quadcopter by generating the desired control signals. The state transition functions (equations (3)) and measurements functions (equations (4)) are used by UKF block to estimate the states of quadcopter.

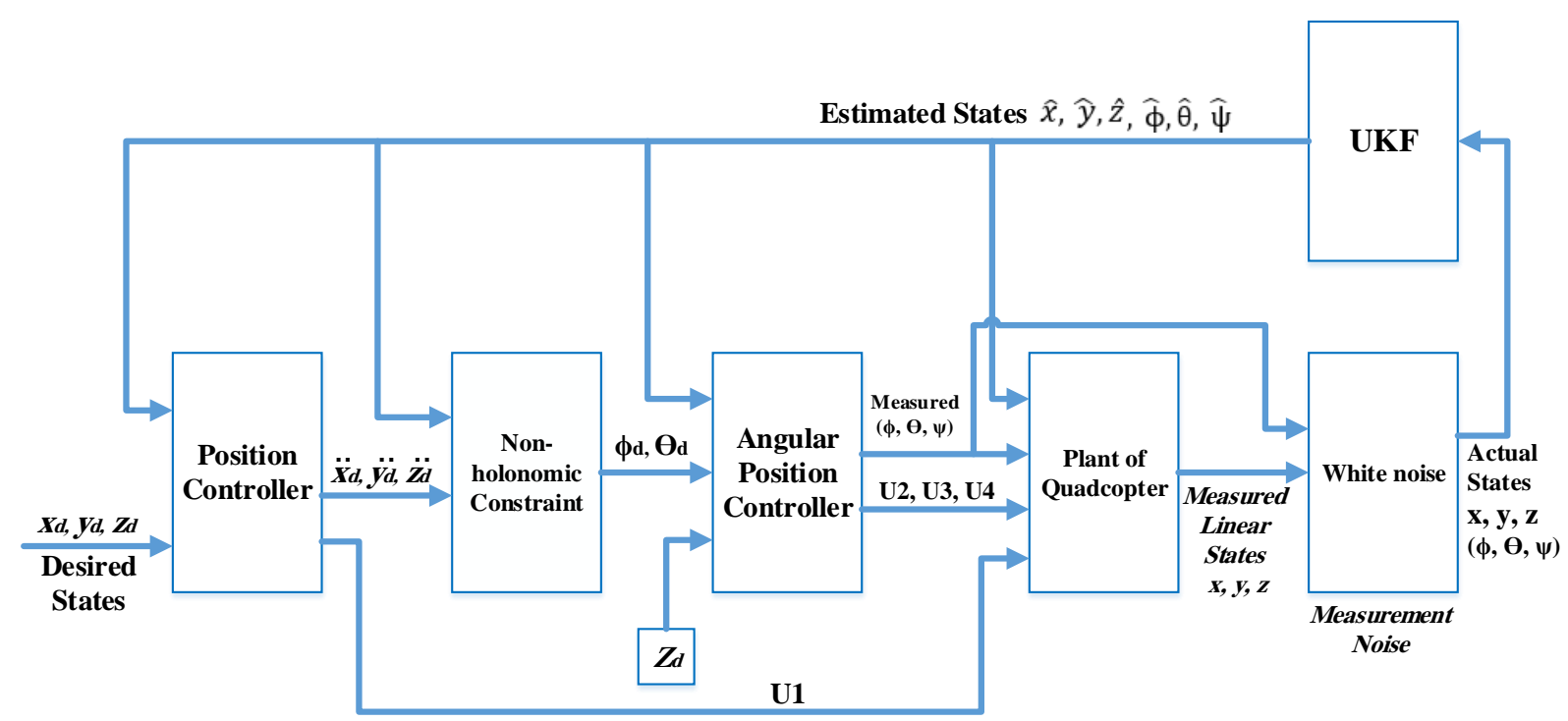

Figure 4. The estimation and control block diagram of the quadrotor

\section{Results and Discussion}

A dynamic model of the quadcopter and UKF are used in our study and performed by using matlab simulink 2018a. Firstly, the mathematical dynamic model of quadcopter with PI-D controller are performed to tuned the gains of PI-D of all states for better response. Table 1 represent the physical parameters of quadcopter while table 2 represent the tuned gains of PI-D controllers.

Table 1. Fixed physical parameters values of the quadcopter

$\begin{array}{clcc}\text { Parameter } & \text { Value } & \text { Parameter } & \text { Value } \\ \mathrm{m} & 0.8 \mathrm{~kg} & \mathrm{l}_{\mathrm{zz}} & 0.02 \mathrm{~kg} \cdot \mathrm{m}^{2} \\ \mathrm{~g} & 9.81 \mathrm{~m} / \mathrm{s}^{2} & \mathrm{k} & 3.13^{*} 10^{-5} \\ 1_{\mathrm{xx}} & 0.015 \mathrm{~kg} \cdot \mathrm{m}^{2} & \mathrm{~d} & 7.5^{*} 10^{-7} \\ 1_{\mathrm{yy}} & 0.015 \mathrm{~kg} \cdot \mathrm{m}^{2} & 1 & 0.25 \mathrm{~m}\end{array}$

Table 2. Tuned values of PI-D controller gains for each state

$\begin{array}{clcc}\text { Parameter } & \text { Value } & \text { Parameter } & \text { Value } \\ \mathrm{K}_{\mathrm{px}} & 2 & \mathrm{~K}_{\mathrm{d} \phi} & 0.5 \\ \mathrm{~K}_{\mathrm{py}} & 2 & \mathrm{~K}_{\mathrm{d} \Theta} & 0.5 \\ \mathrm{~K}_{\mathrm{pz}} & 10 & \mathrm{~K}_{\mathrm{d} \psi} & 0.7997 \\ \mathrm{~K}_{\mathrm{p} \phi} & 1.5 & \mathrm{~K}_{\mathrm{Ix}} & 0.0088\end{array}$




$\begin{array}{clcc}\text { Parameter } & \text { Value } & \text { Parameter } & \text { Value } \\ \mathrm{K}_{\mathrm{p} \Theta} & 1.5 & \mathrm{~K}_{\mathrm{Iy}} & 0.0088 \\ \mathrm{~K}_{\mathrm{p} \psi} & 0.21 & \mathrm{~K}_{\mathrm{Iz}} & 2 \\ \mathrm{~K}_{\mathrm{dx}} & 2.8 & \mathrm{~K}_{\mathrm{I \phi}} & 0.8775 \\ \mathrm{~K}_{\mathrm{dy}} & 2.8 & \mathrm{~K}_{\mathrm{I} \Theta} & 0.8775 \\ \mathrm{~K}_{\mathrm{dz}} & 7 & \mathrm{~K}_{\mathrm{I} \psi} & 0.0123\end{array}$

Figure 6(a, b, c, d, e) shows the response of each state $(X, Y, Z, \phi$, and $\boldsymbol{\theta})$ for constant desired position $(x=12$, $\mathrm{y}=10, \mathrm{z}=5$, and $\psi=0$ ) of quadcopter through 15 seconds. The results are obtained by implementing the block diagram reported in Figure 3.

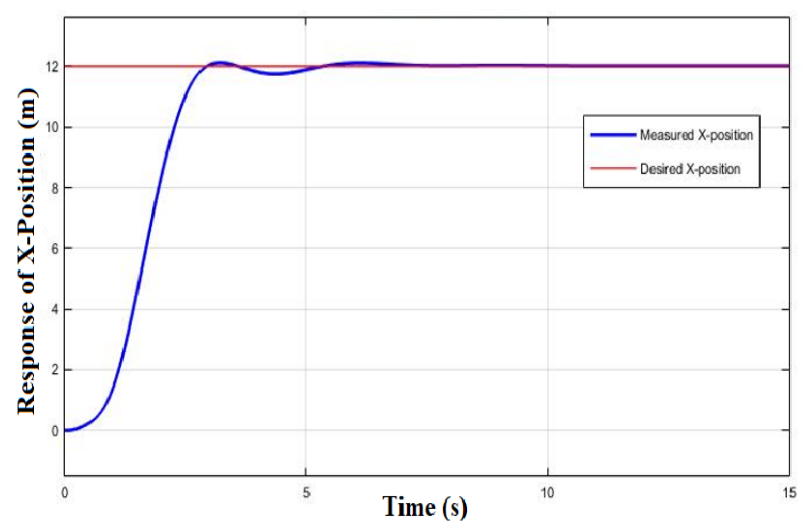

(a)

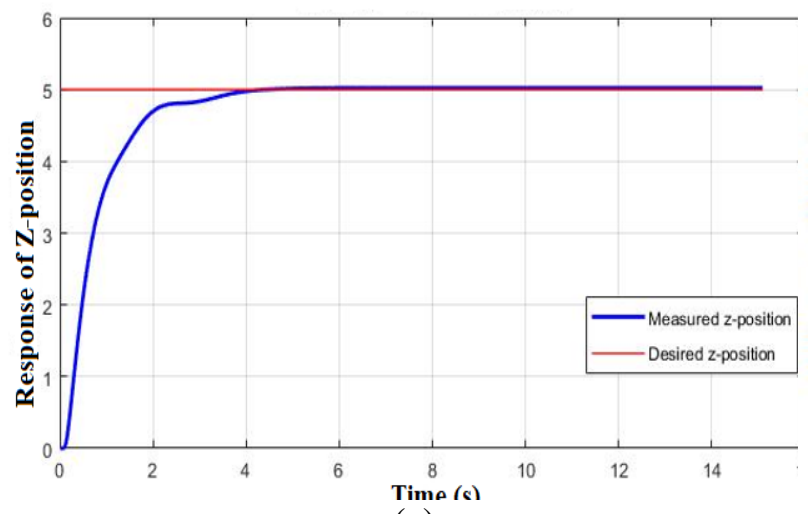

(c)

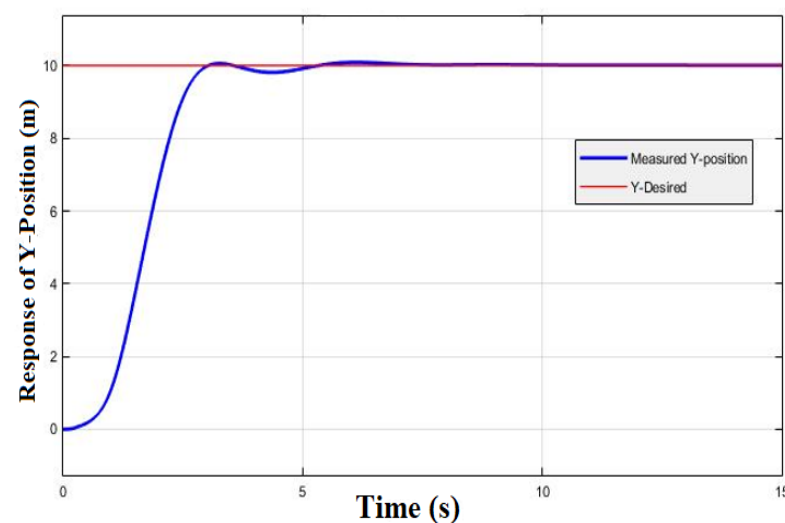

(b)

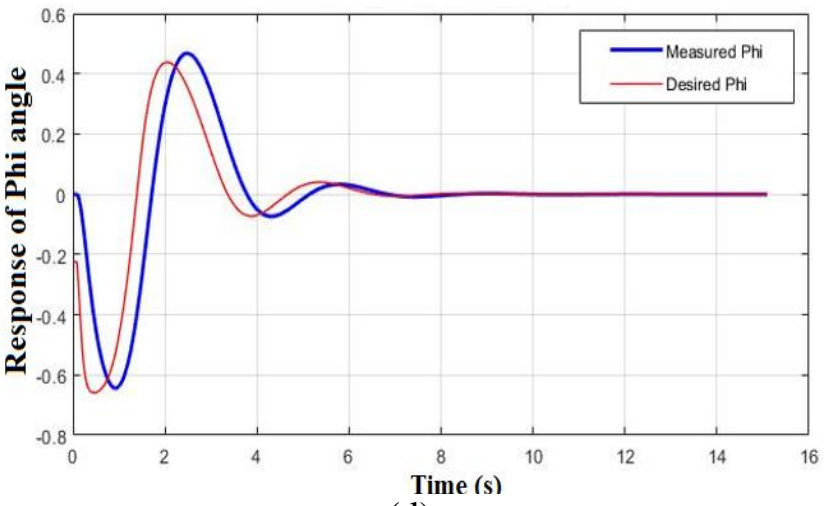

(d)

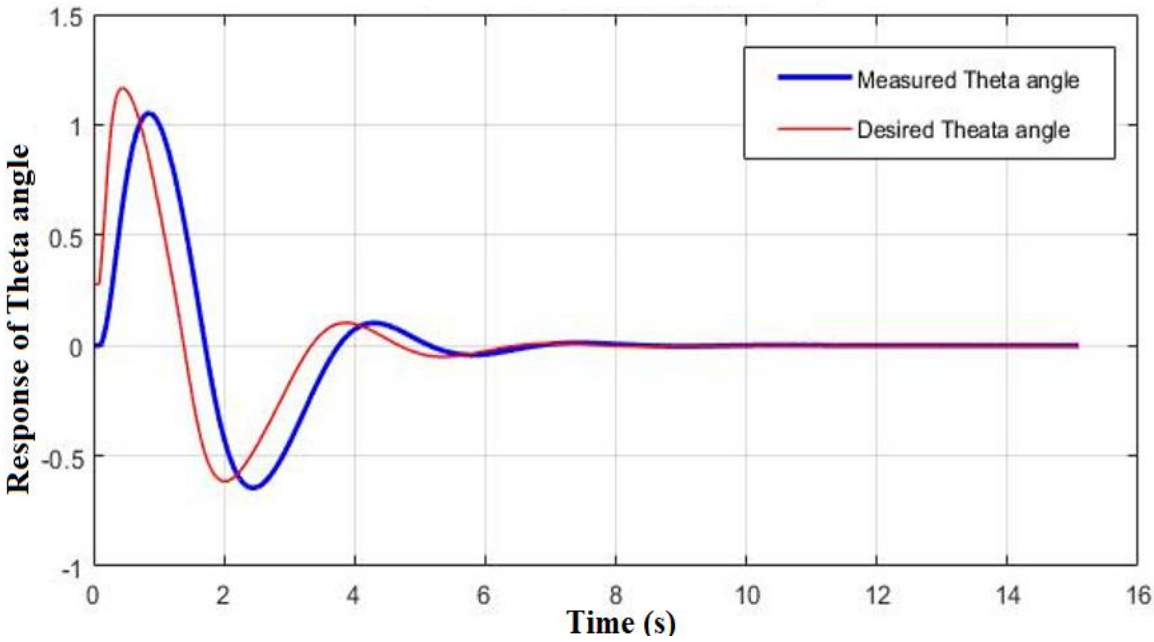

(e)

Figure $6(\mathrm{a}, \mathrm{b}, \mathrm{c}, \mathrm{d}, \mathrm{e})$. Time response of quadcopter states under PI-D controller 
Each quadcopter state needs to have its own PI-D controller to control it. The gains $\left(\mathrm{K}_{\mathrm{p}}, \mathrm{K}_{\mathrm{i}}, \mathrm{K}_{\mathrm{d}}\right)$ of each state controller are tuned manually to get better response as much as possible.

Now, a block diagram in Figure 4 is implemented where white noise is added to each measured state to make simulation more realistic and similar to the real quadcopter. Accurate states are measured by proposed filter where added to the simulation blocks. The measured states have direct influence of quadcopter controller. Therefore, accurate measured states lead to optimized quadcopter performance and increase its stability and resistant against external forces like wind. In the real world, most quadcopter routes are nonlinear so, nonlinear sine wave trajectory with $0.4 \mathrm{rad} / \mathrm{sec}$ and 6 meters' peak-to-peak is used to test our proposed estimator method. The desired altitude is chosen 10 meter so, the quadcopter first takes off to the desired altitude and then follow the desired nonlinear trajectory in the $x-y$ plane for 35 seconds. Figures $7 \mathrm{a}, 7 \mathrm{~b}$, and $7 \mathrm{c}$ shows the response of quadcopter to the nonlinear trajectory. To see how efficiently our proposed method, so it compared with other modern estimation filter called Extended Kalman Filter (EKF) where used by reference (4) for the same purpose in 2018.

Time Response of X-Axis

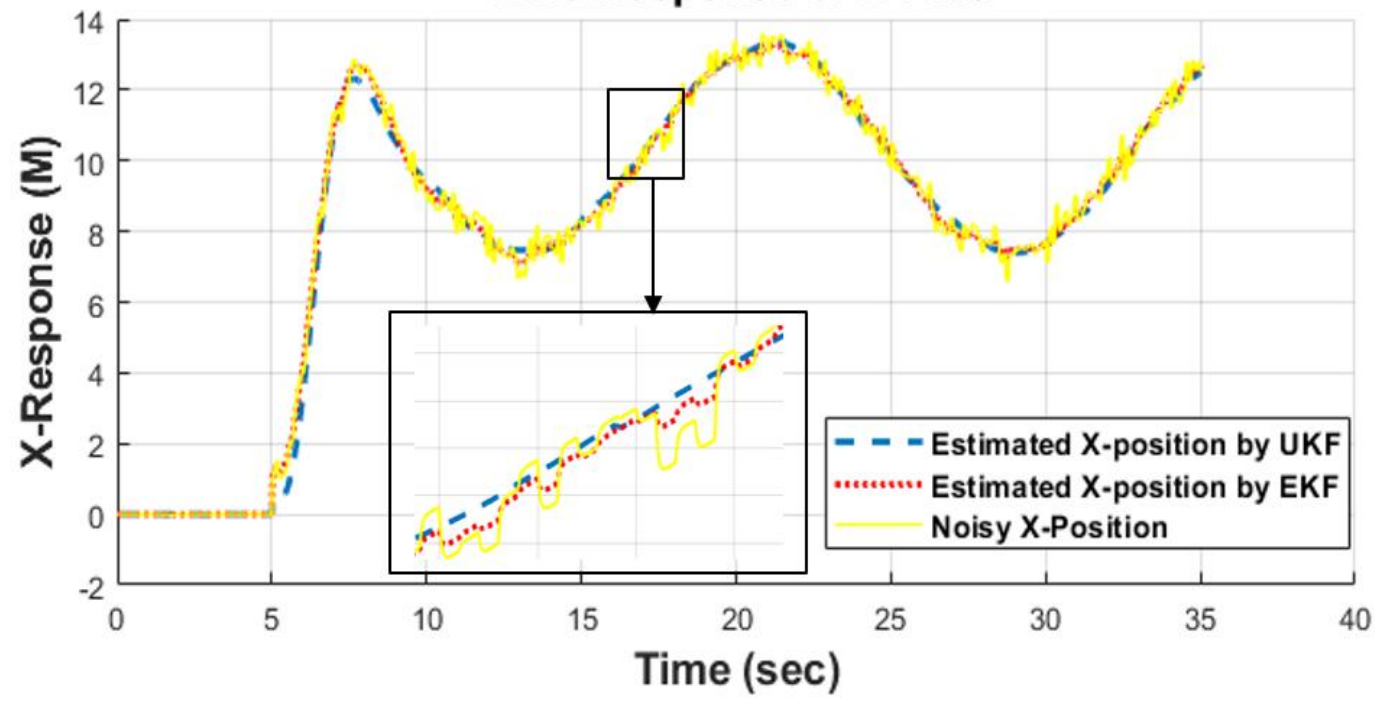

(a)

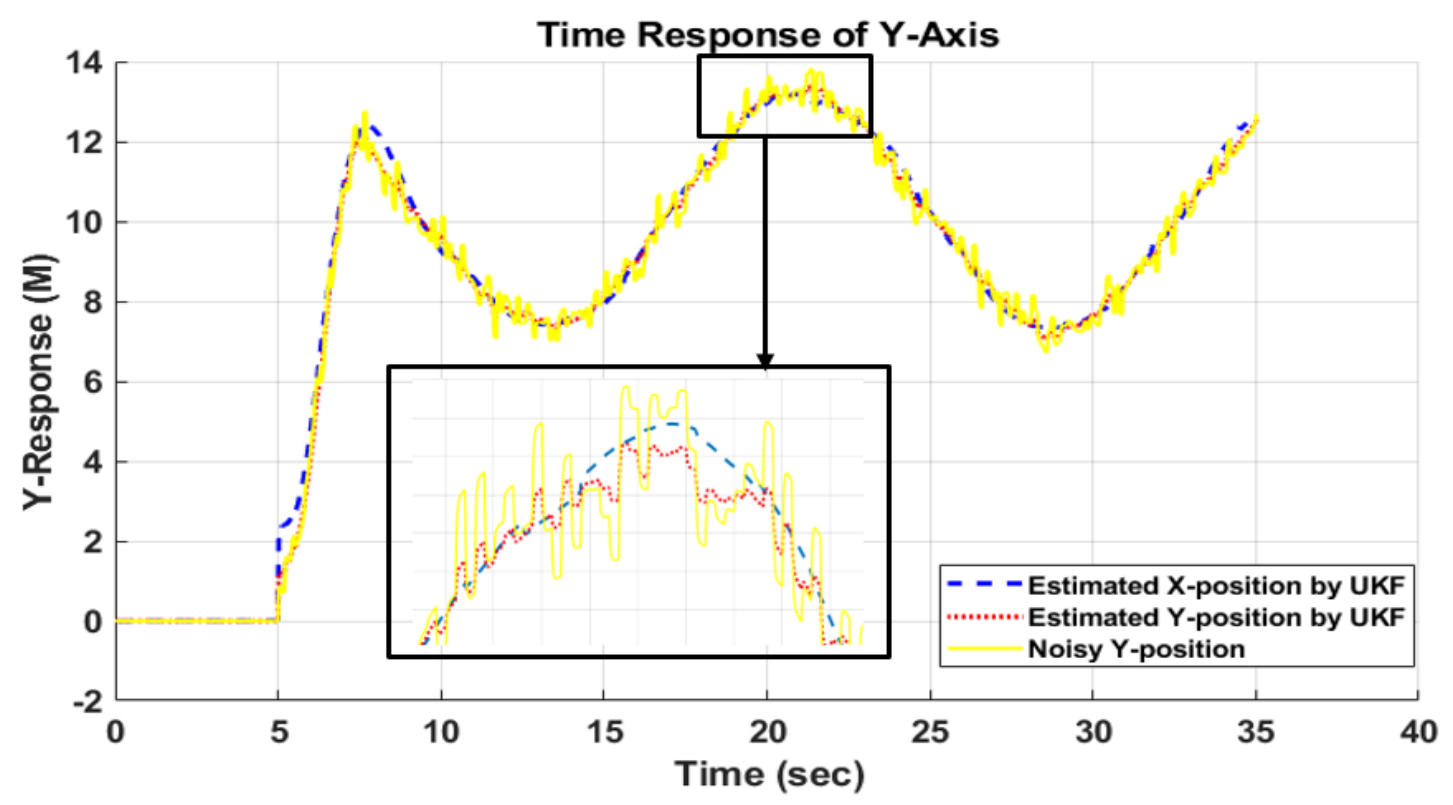

(b) 


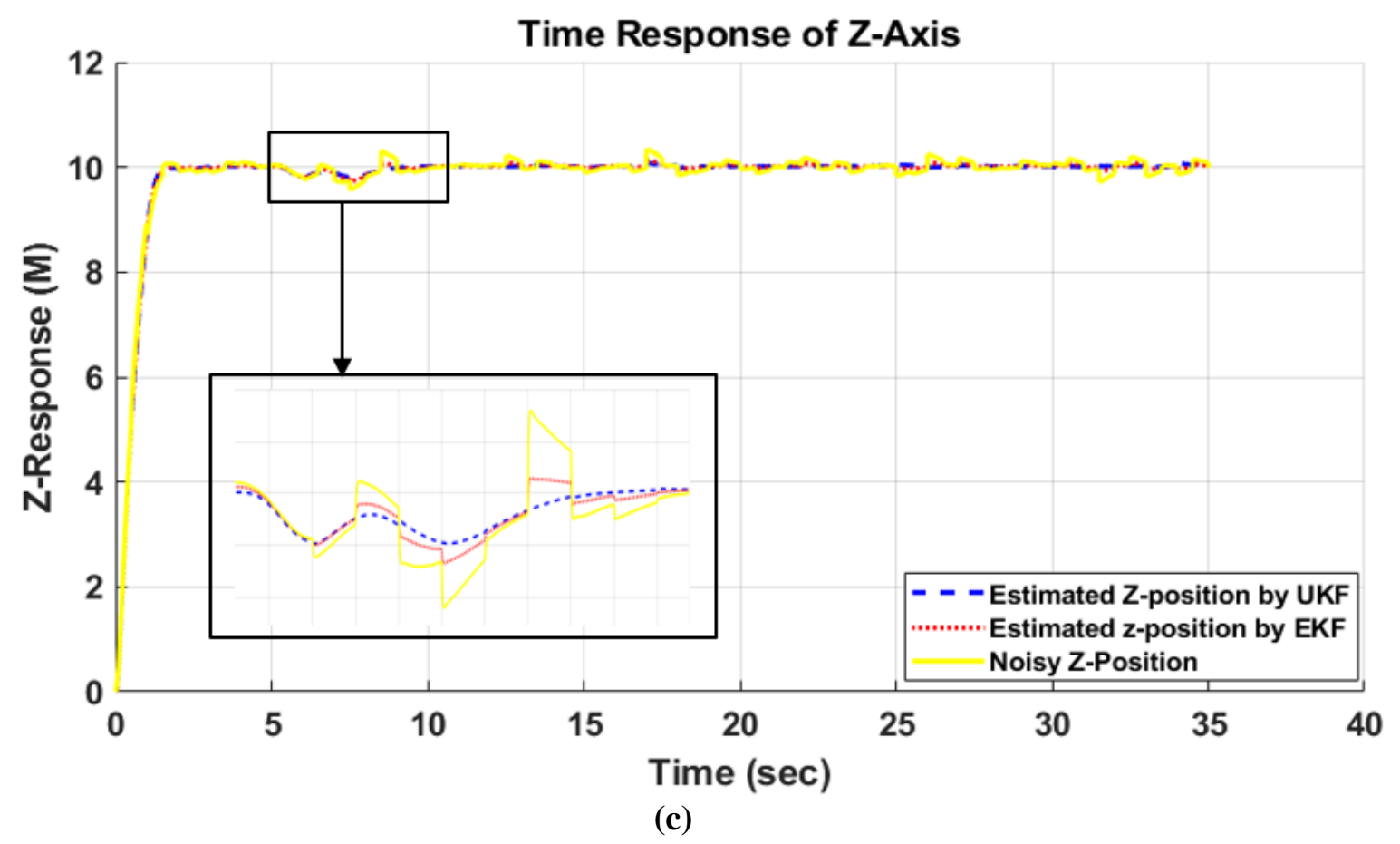

Figure $7(a, b, c) . x, y$, and z-positions responses estimated by UKF and EKF

Figure 7a shows the response of linear x-position of quadcopter to the desired nonlinear sinusoidal path. It is very clear that the estimated response by UKF (Blue line) follow the noisy response signal (Yellow line) with less fluctuations and smoother than the estimated response by EKF (Red line). Also, the estimated y-position (figure 7b) and z-position responses (figure 7c) by UKF are less fluctuations and smoother than the estimated responses by EKF. All results in figures 7 showes that the estimated quadcopter position states by UKF from noisy position signals are more accurate than the states estimated by EKF. Also, the estimated movement of quadcopter to follow the desired trajectory by UKF is smoother and less fluctuations than the estimated movement by EKF. This mean that the UKF is increase the performance of quadcopter and its resistanse to the external desterbances like wind more than the EKF.

\section{Conclusion}

In this paper, we addressed the problem of error in measured states caused by generated noise from physical sensors of quadcopter. Noisy measured states will reduce the performance of quadcopter and decreases its resistance to external disturbances like wind. Different traditional and modern estimation filters are used by researchers to estimate accurate states by decreasing the effect of noise as much as possible. But these filters can't work properly with high nonlinearity system while quadcopter has high nonlinearity behavior. The UKF is proposed in this paper because it is designed to operate properly with high nonlinear system. The results of estimated states by UKF are compared with the states measured by other modern estimation filter called EKF for more reliability. All results show that the states estimated by UKF are more accurate compared with states estimated by EKF. The movements of quadcopter with proposed filter are smother and closer to the desired path. This lead to improving the performance of quadcopter and increases its stability against external disturbances.

\section{References}

[1] Shweta G., Paul I. T. M., James M. Conrad," A Survey of Quadrotor Unmanned Aerial Vehicles", Conference Proceedings of IEEE Southeastcon, 15-18 March 2012.

[2] S. Sarath Chandra, A. S. C. S. Sastry," Autopilot Quadcopter", Periodicals of Engineering and Natural Sciences, Vol.6, No.1, pp. 326-331, December, 2018. 
[3] Johan Jansen," Autonomous Localization and Tracking for UAVs using Kalman Filtering", Master thesis, Norwegian University of Science and Technology, Faculty of Information Technology, Mathematics and Electrical Engineering, July, 2014.

[4] M. R. Mosavi, I. Emam Gholipour," De-noising of GPS Receivers Positioning Data Using Wavelet Transform and Bilateral Filtering", Springer, Wireless Personal Communications Journal, pp. 2295-2312, New York, 2012.

[5] J. A. A. S. Somasiri, D. P. Chandima, A. G. B. P Jayasekara," Extended Kalman Filter Based Autonomous Flying System for Quadcopters", IEEE, 2nd International Conference on Electrical Engineering (EECon), Colombo, Sri Lanka, pp. 130-137, 28 Sep 2018.

[6] Stanislaw K., Piotr K., Jan M.,"Comparison of Estimation Accuracy of EKF, UKF AND PF Filters", WIT Transactions on Modelling and Simulation, VOL. 46, pp. 779-789, 2007.

[7] Zongsheng Zheng, Junbo Zhao, Lamine Mili, Zhigang Liu.” Unscented Kalman Filter-based Unbiased Minimum-Variance Estimation for Nonlinear Systems with Unknown Inputs", IEEE Signal Processing Letters, VOL. 26, NO. 8, pp. 1162-1166, August 2019.

[8] Pengcheng Wang, Zhihong Man, Zhenwei Cao, Jinchuan Zheng, Yong Zhao,” Dynamics Modelling and Linear Control of Quadcopter", IEEE, Proceedings of the International Conference on Advanced Mechatronic Systems, Melbourne, Australia, pp. 498-503 November 30 - December 3, 2016.

[9] Fernando H.C.T.E., De Silva A.T. A., De Zoysa M.D.C., Dilshan K.A.D.C, Munasinghe S.R.," Modelling, Simulation Implementation pf a Quadrotor UAV", IEEE 8th International Conference on Industrial and Information Systems, ICIIS, pp. 207-212, Sri Lanka, August, 2013.

[10] Sevkuthan Kurak, Migdat Hodzic," Control and Estimation of a Quadcopter Dynamical Model", Periodicals of Engineering and Natural Sciences, Vol.6, No.1, pp. 63-75, March, 2018.

[11] M. Rich, "Model development, system identification, and control of a quadrotor helicopter", Graduate 2012 .

Theses and Dissertations, Electrical and Computer Engineering, Paper 12770, Iowa State University,

[12] Sumaila Musa,” Techniques for Quadcopter Modelling \& Design: A Review”. Journal of Unmanned System Technology, Vol. 5, No. 3, pp. 66-75, 2017.

[13] Jose J. Castillo-Zamora, Karla A. Camarillo-Gomez, Gerardo I. Perez-Soto, Juvenal RodriguezResendiz,"Comparison of PD, PID and Sliding-Mode Position Controllers for V-tail Quadcopter Stability",

IEEE Access Journal, Vol. 4, 2018.

[14] V.T. Hoang, M.D. Phung, Q.P. Ha," Adaptive Twisting Sliding Mode Control for Quadrotor Unmanned Aerial Vehicles", IEEE, 11th Asian Control Conference (ASCC), Australia, pp.671-676, December 1720 ,

2017.

[15] Yaser Alaiwi , Aşkın Mutlu," Modelling, Simulation and Implementation of Autonomous Unmanned Quadcopter". Journal of Machines, Technologies and Materials. 2018.

[16] Denis Kotarski, Zoran Benić, Matija Krznar," Control Design for Unmanned Aerial Vehicles with Four Rotors". Journal of Interdisciplinary Description of Complex Systems 14(2), page 236-245, march, 2016.

[17] Stephen Armah, Sun Yi, Wonchang Choi, Dongchul Shin," Feedback Control of Quad-Rotors with a Matlab-Based Simulator". American Journal of Applied Sciences, Vol. 13, Issue 6, pp. 779-793, USA, 
2016.

[18] Bouzgou K., Bestaoui Y., Benchikh L., Ibari B., Ahmed F. Z. Bouzgou K,” Dynamic Modeling, Simulation and PID Controller of Unmanned Aerial Vehicle UAV", IEEE, The Seventh International Conference on Innovative Computing Technology (INTECH), USA,16-18 Aug 2017.

[19] T. Fiorenzani, C. Manes, G. Oriolo, P. Peliti," Comparative Study of Unscented Kalman Filter and Extended Kalman Filter for Position/Attitude Estimation in Unmanned Aerial Vehicles", Istituto di Analisi

Sistemi ed Informatica, IASI-CNR, August, 2008.

[20] Mathieu St-Pierre, Denis Gingras Dr. Ing.," Comparison between the unscented Kalman filter and the extended Kalman filter for the position estimation module of an integrated navigation information system",

IEEE Intelligent Vehicles Symposium Conference, Parma, Italy, pp. 831-835, 14-17 June, 2004.

[21] Eric A. Wan and Rudolph van der Menve,” The Unscented Kalman Filter for Nonlinear Estimation”, In Proceedings of Symposium 2000 on Adaptive Systems for Signal rocessing, Communication and Control(AS-SPCC), IEEE Press.2000.

[22] Simon Julier, Jeffrey Uhlmann, and Hugh F. Durrant-Whyte,” A New Method for the Nonlinear Transformation of Means and Covariances in Filters and Estimators", IEEE Transactions on Automatic Control, VOL. 45, NO. 3, MARCH 2000. 